\title{
Correspondence
}

\section{TNF/TNF-R1 pathway is involved in doxorubicin-induced acute sterile inflammation}

\author{
A Kaczmarek ${ }^{1,2}$, O Krysko ${ }^{3}$, L Heyndrickx ${ }^{1,2}$, T Løve Aaes ${ }^{1,2}$, T Delvaeye ${ }^{1,2,4}$, C Bachert ${ }^{3}$, L Leybaert ${ }^{4}$, P Vandenabeele ${ }^{*, 1,2,5}$ \\ and DV Krysko*,1,2,5
}

Cell Death and Disease (2013) 4, e961; doi:10.1038/cddis.2013.496; published online 12 December 2013

Subject Category: Immunity

\section{Dear Editor,}

Doxorubicin is an anthracycline antibiotic, which is used in treatment of cancer and it works by induction of apoptosis in cancerous cells. ${ }^{1}$ In vivo, apoptotic cells are rapidly cleared preferentially by monocytes and neutrophil migration is inhibited $^{2}$ to limit tissue injury and inflammation. More recently, few anti-cancer drugs, including anthracyclines (doxorubicin) were shown to evoke apoptosis in cancerous cells, which is associated with immune system activation (i.e. immunogenic apoptosis). ${ }^{3,4}$ In our previous study we reported that doxorubicin can induce acute inflammation in peritoneum, which is associated with apoptosis. ${ }^{5}$ Doxorubicin-killed cells could be also a source of damage-associated molecular patterns (DAMPs) leading to inflammation and tumor necrosis factor (TNF) production, which would amplify the inflammatory response triggered by DAMPs. TNF is a pleiotropic cytokine that binds to receptors TNF-R1 and TNF-R2 and, depending on cell type, triggers different signaling pathways, including cell death and inflammation. ${ }^{6}$ This study aimed to examine whether TNF contributes to the doxorubicin-induced acute sterile inflammation.

Intraperitoneal injection of doxorubicin provoked an acute inflammatory response accompanied by the influx of neutrophils. ${ }^{5}$ We observed an increased level of LDH, a marker of tissue damage and secondary necrotic cells, already after $6 \mathrm{~h}$ with further increase after $16 \mathrm{~h}$ post injection of doxorubicin (Supplementary Figure 1a). Lavage fluid collected $16 \mathrm{~h}$ after doxorubicin injection contained increased levels of TNF as compared to vehicle-injected group $(0.09 \pm 0.14$ versus $2.11 \pm 0.84 \mathrm{pg} / \mathrm{ml}, P<0.0001)$.

We next tested the involvement of TNF-R1 and TNF-R2 in the sterile inflammation in response to doxorubicin. Wild-type mice had abundant neutrophils in their abdominal cavities, but this response was markedly decreased in TNF-R1/2 double knockout mice (by 2.4-fold, Supplementary Figure 1b). The number of neutrophils attracted in response to doxorubicin-induced acute inflammation was 3.4-fold lower in TNF-R1-deficient mice than in controls (Figure 1a), but in TNF-R2-deficient mice, there was no difference as compared to wild-type mice (Figure 1b). These results show that TNF-R1 was involved in mediating the inflammatory response but TNF-R2 was not. Importantly, cell death in TNF-R1/2 double knockout mice was not affected in comparison to wild-type mice (data not shown) indicating that TNF does not contribute to the cell death process itself.

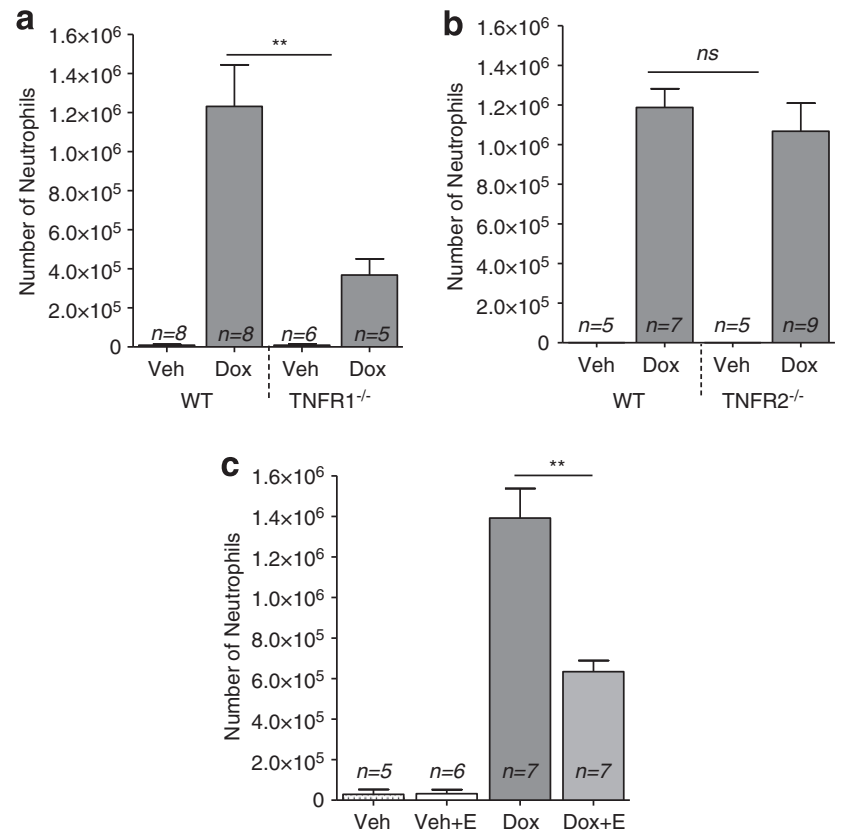

Figure 1 Role of TNF-R1 and TNF-R2 and the effect of inhibiting soluble TNF on doxorubicin-induced acute inflammatory response. The number of neutrophils in peritoneal lavage was decreased in TNF-R1 ${ }^{-l-}$ (a) but not in TNF-R2 ${ }^{-1}$ (b). Treatment of wild-type mice with Etanercept significantly reduced the recruitment of neutrophils $16 \mathrm{~h}$ after intraperitoneal injection of doxorubicin $(\mathrm{Dox}+\mathrm{E})(\mathbf{c})$. Negative controls were injected with vehicle alone or with vehicle plus Enanercept $(V e h+E)$. Means and S.E.M. are for pooled data, and $n$ is the number of mice in each group. ${ }^{* *} P<0.002$. Mann-Whitney test was used to evaluate differences between groups (GraphPad Prism-5). The number of monocytes/macrophages and neutrophils in the peritoneal exudate cells was determined as described previously. ${ }^{5}$

\footnotetext{
${ }^{1}$ Inflammation Research Center, VIB, Ghent, Belgium; ${ }^{2}$ Department of Biomedical Molecular Biology, Ghent University, Ghent, Belgium; ${ }^{3}$ Upper Airway Research Laboratory, Department of Oto-Rhino-Laryngology, Ghent University Hospital, Ghent, Belgium and ${ }^{4}$ Department of Basic Medical Sciences, Physiology Group, Ghent University, Ghent, Belgium

*Corresponding authors: P Vandenabeele or DV Krysko, Inflammation Research Center, VIB-Ghent University, Technologiepark 927 B-9052, Ghent (Zwijnaarde), Belgium. Tel: +32 93313760 (PV) or +32 93313712 (DVK); Fax: +32 9 3313609; E-mail: Peter.Vandenabeele@irc.vib-UGent.be or Dmitri.Krysko@irc.vib-UGent.be ${ }^{5}$ These authors contributed equally to the work.
} 
Further, we investigated whether acute inflammation can be reduced in wild-type mice by the administration of Etanercept, a recombinant dimeric soluble form of TNF-R2 that blocks the interaction of TNF with its cell surface receptors. ${ }^{7}$ Wild-type mice were injected intraperitoneally with Etanercept together with doxorubicin and $16 \mathrm{~h}$ later the recruited cells were phenotyped. Etanercept significantly reduced the recruitment of neutrophils (Figure 1c). This further confirms the importance of TNF in doxorubicininduced acute inflammation.

In conclusion, we report that intraperitoneal injection of doxorubicin in mice leads to increase in TNF levels in the lavage fluid. It has been also reported that patients undergoing chemotherapy with doxorubicin had elevated levels of TNF in their plasma. ${ }^{8}$ Similarly, mouse in vitro and in vivo studies demonstrated up-regulation of TNF after doxorubicin administration both on mRNA and protein levels. ${ }^{9,10}$ Previously, we found that the majority of cells that died apoptotically due to doxorubicin treatment were monocytes/macrophages with some minor neutrophils. ${ }^{5}$ Therefore, it is possible that either dying macrophages or attracted neutrophils might be a source of TNF, and that by binding to TNF-R1 it could amplify the inflammation. We demonstrated that the acute inflammatory response to doxorubicin was reduced in TNF-R1- but not TNF-R2-deficient mice. In addition, Etanercept decreases the attraction of neutrophils after doxorubicin administration. These studies show that TNF and the TNF-R1 signaling pathway are key elements in the acute sterile inflammatory response to doxorubicin.

\section{Conflict of Interest}

The authors declare no conflict of interest.

Acknowledgements. We thank Dr. A Bredan for editing the manuscript. This work was supported by project grants from the Fund for Scientific Research Flanders (FWO-Vlaanderen, G.0728.10, G060713N, G0A5413N to DVK; $3 G 067512$ to DVK and OK) and by an individual research grant from FWOVlaanderen (31507110 to DVK). DVK is a senior postdoctoral researcher paid by a fellowship from FWO-Vlaanderen. AK is a recipient of an Emmanuel van der Schueren scholarship from the Flemish League Against Cancer. Vandenabeele's group is supported by VIB, Ghent University (GROUP-ID Consortium of the UGent MRP initiative), FWO-Vlaanderen (G.0875.11, G.0973.11, G.0A45.12N), Federal Research Program (IAP 7/32), PV holds a Methusalem grant (BOF09/01M00709) from the Flemish Government.

1. Wang S et al. J Biol Chem 2004; 279: 25535-25543.

2. Bournazou I et al. J Clin Invest 2009; 119: 20-32.

3. Apetoh L et al. Trends Mol Med 2008; 14: 141-151.

4. Krysko DV et al. Nat Rev Cancer 2012; 12: 860-875.

5. Krysko DV et al. Cell Death Differ 2011; 18: 1316-1325

6. Balkwill F. Nat Rev Cancer 2009; 9: 361-371.

7. Fantuzzi $\mathrm{F}$ et al. Expert Opin Ther Targets 2008; 12: 1085-1096.

8. Aluise CD et al. Free Radic Biol Med 2011; 50: 1630-1638.

9. Niiya $\mathrm{M}$ et al. Cancer Chemother Pharmacol 2003; 52: 391-398.

10. Nozaki N et al. Circulation 2004; 110: 2869-2874.

(c) (i) $(9)($ Cell Death and Disease is an open-access journal published by Nature Publishing Group. This work is licensed under a Creative Commons Attribution-NonCommercialNoDerivs 3.0 Unported License. To view a copy of this license, visit http://creativecommons.org/licenses/by-nc-nd/3.0/

Supplementary Information accompanies this paper on Cell Death and Disease website (http://www.nature.com/cddis) 